\title{
Analysis of Risk Factors for Colonic Diverticular Bleeding: A Matched Case-Control Study
}

Yuusaku Sugihara ${ }^{*}{ }^{\dagger}$, Shin-ei Kudo*, Hideyuki Miyachi*, Masashi Misawa*, Shogo Okoshi*, Hiroyuki Okada ${ }^{\dagger}$, and Kazuhide Yamamoto ${ }^{\dagger}$

"Digestive Disease Center, Showa University Northern Yokohama Hospital, Yokohama, and ${ }^{\dagger}$ Department of Gastroenterology and Hepatology, Okayama University Graduate School of Medicine, Okayama, Japan

Background/Aims: Diverticular bleeding can occasionally cause massive bleeding that requires urgent colonoscopy (CS) and treatment. The aim of this study was to identify significant risk factors for colonic diverticular hemorrhage. Methods: Between January 2009 and December 2012, 26,602 patients underwent CS at our institution. One hundred twenty-three patients underwent an urgent CS due to acute lower gastrointestinal hemorrhage. Seventy-two patients were diagnosed with colonic diverticular hemorrhage. One hundred forty-nine age- and sex-matched controls were selected from the patients with nonbleeding diverticula who underwent CS during the same period. The relationship of risk factors to diverticular bleeding was compared between the cases and controls. Results: Uni- and multivariate conditional logistic regression analyses demonstrated that the use of nonsteroidal anti-inflammatory drugs (odds ratio [OR], 14.70; 95\% confidence interval [CI], 3.89 to 55.80; $p<0.0001$ ), as well as the presence of cerebrovascular disease (OR, 8.66; 95\% $\mathrm{Cl}, 2.33$ to 32.10; $\mathrm{p}=0.00126$ ), and hyperuricemia (OR, 15.5; 95\% $\mathrm{Cl}, 1.74$ to $138.00 ; \mathrm{p}=0.014$ ) remained statistically significant predictors of diverticular bleeding. Conclusions: Nonsteroidal anti-inflammatory drugs, cerebrovascular disease and hyperuricemia were significant risks for colonic diverticular hemorrhage. The knowledge obtained from this study may provide some insight into the diagnostic process for patients with lower gastrointestinal bleeding. (Gut Liver 2016;10:244-249)

Key Words: Diverticular bleeding; Colonoscopy; Anti-inflammatory agents, non-steroidal; Hyperuricemia; Cerebrovascular disease

\section{INTRODUCTION}

Colonic diverticula are small pouches from the colonic lumen. It typically forms in parallel rows between the taeniae coli because of weakness of the muscle wall at sites of penetration of the vascular supplying the mucosa. ${ }^{1}$ Diverticulosis has been labeled a disease of Western civilization because of its striking geographic variability. The disorder is rare in the countryside of Africa and Asia, with the highest prevalence seen in the United States, Europe, and Australia. ${ }^{2}$

The prevalence of diverticular diseases increases with age in general. ${ }^{3}$ Less than 10\% of cases are in adults aged 40 years or more. However, almost one-third of the people aged up to 50 years have diverticulosis, and up to $60 \%$ of people older than 80 years have been affected. ${ }^{4}$ In Japan, the prevalence of diverticular disease among the aged population also has increased as a result of insufficient dietary fiber intake, as in Western countries. $^{5}$

Around 75\% to $80 \%$ of patients with anatomical diverticulosis will remain asymptomatic throughout their lifetime. ${ }^{1}$ However, a few develop complications such as diverticulitis, abscesses, fistulas, obstruction, and hemorrhage. One of the most frequent complications is diverticular hemorrhage.

Diverticular hemorrhage is one of the common causes of lower gastrointestinal bleeding and accounts for $41 \%$ of gastrointestinal bleeding. ${ }^{6}$ Severe hemorrhage can arise in 3\% to 5\% of patients with diverticulosis and can frequently cause massive bleeding that requires urgent colonoscopy (CS).

In pathology, the microangiography of resected specimens from patients with bleeding diverticula typically shows intimal thickening and medial thinning of the vasa recta as it runs over the dome of the diverticulum. ${ }^{7}$ These changes arise asymmetri-

Correspondence to: Yuusaku Sugihara

Digestive Disease Center, Showa University Northern Yokohama Hospital, 35-1 Chigasaki-chuo, Tsuzuki-ku, Yokohama 224-8503, Japan

Tel: +81-045-949-7000, Fax: +81-045-949-7263, E-mail: y.sugi117@gmail.com

Received on October 22, 2014. Revised on January 12, 2015. Accepted on January 19, 2015. Published online June 19, 2015

pISSN 1976-2283 eISSN 2005-1212 http://dx.doi.org/10.5009/gnl14407

(a) This is an Open Access article distributed under the terms of the Creative Commons Attribution Non-Commercial License (http://creativecommons.org/licenses/by-nc/4.0) which permits unrestricted non-commercial use, distribution, and reproduction in any medium, provided the original work is properly cited. 
cally toward the lumen, leading to a segmental weakening of the artery and eventually rupture. The factors that initiate these arterial changes are unknown, although inflammation does not seem to be a contributing factor. ${ }^{1}$ This finding is in agreement with the clinical with the clinical impression that bleeding rarely complicates diverticulitis.

Till date, several reports have analyzed the background factors that might be associated with diverticular hemorrhage. ${ }^{8}$ However, to the best of our knowledge there have been few reports that have performed analysis in the form of a matched case- control study at a high volume center.

The aim of the present study is to confirm the etiologic factors that cause diverticular hemorrhage by analyzing patients who were cared for at a single center.

\section{MATERIALS AND METHODS}

\section{Patients and methods}

Between January 2009 and December 2012, 26,602 patients underwent a CS at Showa University Northern Yokohama Hospital, Yokohama, Japan. One hundred twenty-three Japanese patients had an urgent CS due to acute lower gastrointestinal hemorrhage. Patients with iatrogenic hemorrhages, caused by endoscopic mucosal resection and endoscopic submucosal dissection, were excluded. Our criterion for performing urgent CS was that the procedure was undertaken within 24 hours of visiting our institution for the diagnosis and treatment of lower gastrointestinal hemorrhage. Of the 123 patients, 72 patients were diagnosed with colonic diverticular hemorrhage on the basis of the following criteria, which was based on the report by Jensen et al.: ${ }^{9}$ active bleeding from a diverticulum was observed or blood coagulates were found in the colon with diverticula in the absence of blood in the terminal ileum and any other demonstrable cause of bleeding. When the urgent CS detected an active bleeding point at a diverticulum, endoscopic hemostasis with a hemoclip was performed.

We selected 149 age- and sex-matched controls from the nonbleeding diverticulum patients who underwent CS for other reasons during the same period. These controls were diagnosed with colonic diverticulosis by CS, and they had not experienced bleeding (Fig. 1).

To obtain accurate information, we administrated a questionnaire at the time of CS at our endoscopic unit by medical research staff. The staff double-checked the medical records to avoid possible miscalculations. The information included the presence of cerebrovascular disease (cerebral infarction or hemorrhage), ischemic heart disease, hypertension, hyperlipidemia, diabetes mellitus, chronic renal disease, osteoporosis, and hyperuricemia. These diseases were considered present if the patients had been diagnosed with the condition in past or were taking medicine to treat the condition at the time of investigation. Moreover, information was gathered about medications, including nonsteroidal anti-inflammatory drugs (NSAIDs), antithrombotic agents (antiplatelets, anticoagulants, and low-dose aspirin), body mass index, and lifestyle factors such as alcohol consumption was gathered. We obtained the information about the type of NSAIDs, such as coxib- or noncoxib agents and about the frequency of usage, such as regular or intermittent intake. Subjects who consumed more than $350 \mathrm{~mL}$ beer considered to be drinkers, and those who smoked more than 10 cigarettes per day were considered to be smokers. The relationship of these factors with diverticular bleeding was compared between cases and controls. The Ethics Committee of the Showa University Northern Yokohama Hospital approved this study protocol.

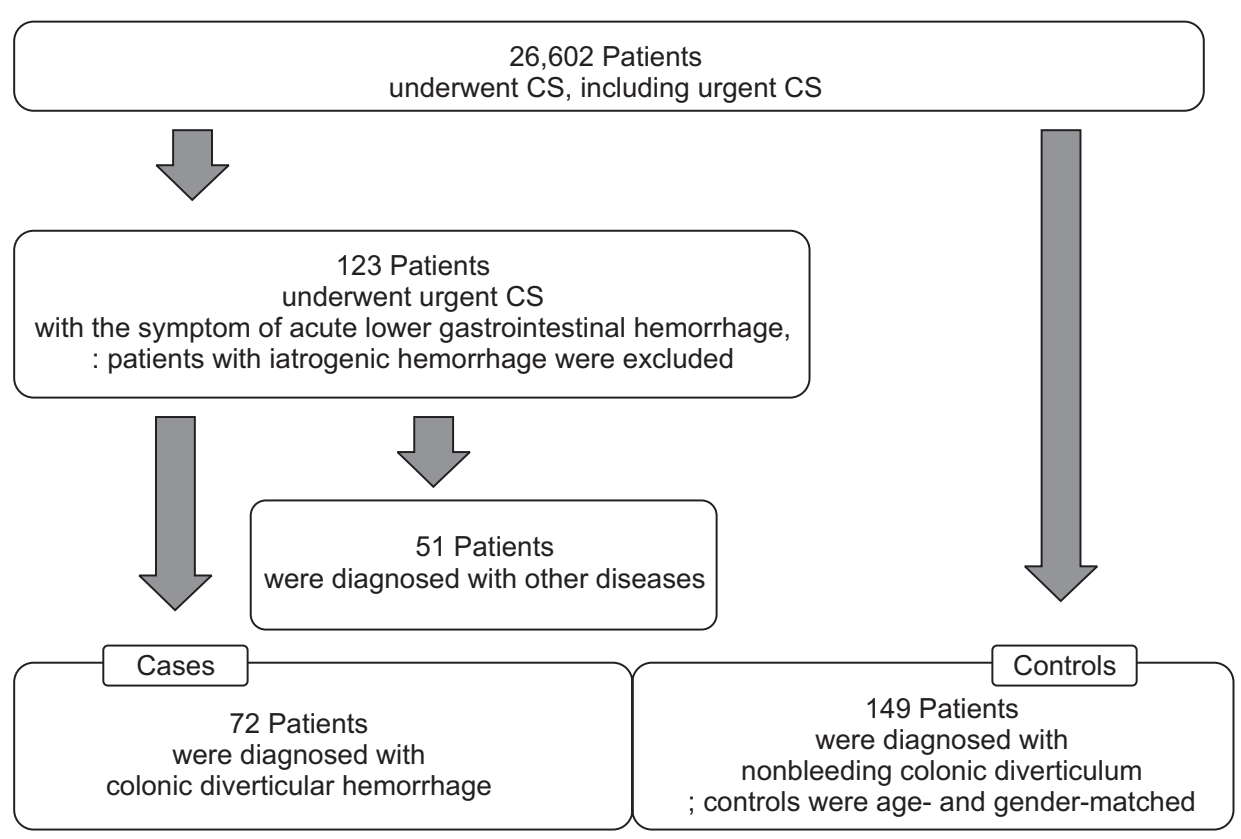

Fig. 1. Clinical data regarding the patients enrolled in the present study. CS, colonoscopy. 


\section{Statistical analysis}

To estimate the risk factors for diverticular bleeding, the factors were compared between cases and controls. The data were evaluated using descriptive statistical methods (means \pm standard deviations). For the comparison of two factors, the unpaired Student t-test was used. A frequency distribution was calculated by the chi-square test or Fisher exact probability test. Univariate and multivariate conditional logistic regression analyses procedures were used to obtain crude and adjusted odds ratios (ORs) and 95\% confidence intervals (95\% CI) after controlling simultaneously for potential confounders. Two-sided pvalues less than 0.05 were regarded as statistically significant. Multicollinearity was assessed by the variance inflation factor. A variance inflation factor was regarded as indicating serious multicollinearity, and values greater than 2.0 may be a cause for

Table 1. Source of Acute Lower Gastrointestinal Bleeding

\begin{tabular}{lccc}
\hline \multicolumn{1}{c}{ Disease } & No. & $\%$ & Male/female \\
\hline Diverticular hemorrhage & 72 & 58.5 & $51 / 21$ \\
Ileum hemorrhage & 8 & 6.5 & $5 / 3$ \\
Ischemic colitis & 7 & 5.7 & $4 / 3$ \\
Inflammatory bowel disease & 6 & 4.9 & $5 / 1$ \\
Hemorrhoids & 5 & 4.1 & $3 / 2$ \\
Infection colitis & 4 & 3.3 & $2 / 2$ \\
Anastomotic hemorrhage & 4 & 3.3 & $2 / 2$ \\
Solitary rectal ulcer & 3 & 2.4 & $2 / 3$ \\
Carcinoma & 3 & 2.4 & $2 / 2$ \\
Sigmoid volvulus & 3 & 2.4 & $2 / 0$ \\
Unknown & 4 & 3.3 & $2 / 2$ \\
Others & 4 & 3.3 & $2 / 2$ \\
Total & 123 & & $80 / 43$ \\
\hline
\end{tabular}

concern. All analyses were performed using R version 2.14.0. ${ }^{10}$

\section{RESULTS}

Table 1 shows the diagnoses of the patients with acute lower gastrointestinal bleeding. Diverticular hemorrhage was the most frequent cause of lower gastrointestinal hemorrhage, and it accounted for 72 of 123 (58.5\%) of all cases of acute bleeding from the lower intestine. Twenty-six patients were diagnosed with diverticular hemorrhage because of active bleeding from a diverticulum, and 46 patients were diagnosed because of observing blood coagulates in the diverticula in the absence of blood in the terminal ileum and any other demonstrable cause of bleeding.

In the patients with diverticular hemorrhage, 22 patients (31\%) had severe anemia that needed red cell transfusions of two units or more. During the preparation for the urgent CS, 59 patients (82\%) had been given polyethylene glycol (PEG), 11 patients (15\%) had been administrated by a glycerin enema (GE), and two patients (3\%) were received nothing (no-prep). We detected the 26 bleeding points $(26 / 72,36 \%)$ for these diverticular hem-

Table 2. Background Factors of the Cases and Controls

\begin{tabular}{lccc}
\hline \multicolumn{1}{c}{ Factor } & Cases & Controls & p-value \\
\hline No. & 72 & 149 & - \\
Male/female & $51 / 21$ & $91 / 58$ & - \\
Average age (range), yr & $70(33-92)$ & $70(37-92)$ & - \\
Location of diverticula (L/R) & $24 / 48$ & $77 / 72$ & - \\
Body mass index, kg/m ${ }^{2}$ & 21.3 & 22.8 & NS \\
Smoking & 10 & 18 & NS \\
Alcohol & 14 & 40 & NS
\end{tabular}

L, left hemicolon; $\mathrm{R}$, right hemicolon; NS, not significant.

Table 3. Risk Factors for Diverticular Hemorrhage by Univariate Analysis

\begin{tabular}{|c|c|c|c|c|c|}
\hline Factor & $\begin{array}{l}\text { Cases } \\
(n=72)\end{array}$ & $\begin{array}{c}\text { Controls } \\
(n=149)\end{array}$ & OR & $95 \% \mathrm{CI}$ & p-value \\
\hline \multicolumn{6}{|l|}{ Past history } \\
\hline Diabetes mellitus & 14 & 18 & 1.398 & $0.556-3.389$ & 0.5177 \\
\hline Hypertension & 36 & 42 & 2.399 & $1.287-4.498$ & 0.0041 \\
\hline Hyperlipidemia & 6 & 10 & 1.262 & $0.361-4.026$ & 0.7825 \\
\hline Ischemic heart disease & 17 & 14 & 2.964 & $1.277-6.993$ & 0.0067 \\
\hline Cerebrovascular disease & 14 & 4 & 2.325 & $0.682-3.410$ & 0.0006 \\
\hline Chronic renal disease & 6 & 7 & 1.839 & $0.489-6.672$ & 0.3606 \\
\hline Osteoporosis & 1 & 2 & 2.092 & $0.149-29.412$ & 0.5978 \\
\hline Hyperuricemia & 8 & 1 & 18.266 & $2.368-822.732$ & 0.0006 \\
\hline \multicolumn{6}{|l|}{ Medication } \\
\hline NSAIDs & 19 & 3 & 17.194 & $4.786-94.425$ & $<0.0001$ \\
\hline Antithrombotic agents & 29 & 20 & 4.316 & $2.120-8.964$ & $<0.0001$ \\
\hline
\end{tabular}

OR, odds ratio; CI, confidence interval; NSAIDs, nonsteroidal anti-inflammatory drugs. 
orrhages; in 19 of the 59 PEG cases (32\%), six of the $11 \mathrm{GE}$ cases (55\%), and one of the two no-prep cases (50\%). Table 2 shows the clinical characteristics of the cases and controls. The age range of the patients was 33 to 93 years (average, 70 years). The male:female sex ratio of the patients was 51:21. Twentyfour diverticula were located in the left hemicolon and 48 in the right hemicolon in the patients, whereas 77 were located in the left hemicolon, and 72 in the right hemicolon in the controls. The body mass index was 21.3 in the cases and 22.8 in the controls. Drinkers and smokers habits were recognized to be 14 $(19.4 \%)$ and $10(13.9 \%)$ of the bleeding cases, and $40(26.8 \%)$ and 14 (9.3\%) of the controls, respectively.

By using age and sex as matching variables, we conducted a

Table 4. Risk Factors for Diverticular Hemorrhage by Multivariate Analysis

\begin{tabular}{lclc}
\hline \multicolumn{1}{c}{ Factor } & OR & \multicolumn{1}{c}{$95 \% \mathrm{CI}$} & $\mathrm{p}$-value \\
\hline Hypertension & 1.56 & $0.760-3.190$ & 0.226 \\
Ischemic heart disease & 1.64 & $0.496-5.430$ & 0.418 \\
Cerebrovascular disease & 8.66 & $2.330-32.100$ & 0.00126 \\
Hyperuricemia & 15.5 & $1.740-138.000$ & 0.014 \\
NSAIDs & 14.70 & $3.890-55.800$ & $<0.0001$ \\
Antithrombotic agents & 1.32 & $0.463-3.750$ & 0.604 \\
\hline
\end{tabular}

OR, odds ratio; CI, confidence interval; NSAIDs, nonsteroidal antiinflammatory drugs. case-control study to analyze the risk factors. In the results of the univariate analysis, the factors of cerebrovascular disease, hypertension, hyperuricemia, chronic renal disease, and the usage of antithrombotic agents and NSAIDs were significantly related to an increase in ORs (Table 3). The variance inflation factors for cerebrovascular disease, hypertension, hyperuricemia, chronic renal disease, and the usage of antithrombotic agents and NSAIDs were 1.6946, 1.0671, 1.5757, 1.0166, 1.0694, and 1.1641, respectively. Evidence for multicollinearity was absent because the variance inflation factor for independent variables in all factors was less than 2.0. Using these factors, we performed multivariate conditional logistic regression with stepwise variable selection. Finally, the current usage of NSAIDs (OR, 14.70; 95\% CI, 3.89 to 55.80; $\mathrm{p}<0.0001$ ), the presence of cerebrovascular disease (OR, 8.66; 95\% CI, 2.33 to 32.10; $\mathrm{p}=0.00126)$, and hyperuricemia (OR, 15.5; 95\% CI, 1.74 to $138.00 ; p=0.014$ ) remained statistically significant (Table 4).

Seventy-two patients were diagnosed with a diverticular bleeding by urgent CS. We divided diverticular hemorrhage patients into a definite group (active bleeding from a diverticulum was observed) and a presumptive group (blood coagulates were found in the colon), and reconducted the same case-control study. In the definite group, 26 patients were diagnosed with diverticular hemorrhage with active bleeding, and in the presumptive group, 46 patients with blood coagulate were found in the colon. Table 5 showed the results of the case-control study

Table 5. Risk Factors for Diverticular Hemorrhage in the Definite Group

\begin{tabular}{|c|c|c|c|c|c|}
\hline Factor & Cases & Controls & OR & $95 \% \mathrm{CI}$ & p-value \\
\hline \multicolumn{6}{|c|}{ Univariate analysis in the cases and controls of the definite group } \\
\hline Past history & 26 & 54 & & & \\
\hline Diabetes mellitus & 5 & 7 & 1.599 & $0.454-5.623$ & 0.4647 \\
\hline Hypertension & 15 & 13 & 4.301 & $1.587-11.659$ & 0.0041 \\
\hline Hyperlipidemia & 3 & 4 & 1.631 & $0.337-7.887$ & 0.5433 \\
\hline Ischemic heart disease & 8 & 4 & 5.556 & $1.491-20.703$ & 0.0106 \\
\hline Cerebrovascular disease & 5 & 2 & 6.191 & $1.112-34.444$ & 0.0373 \\
\hline Chronic renal disease & 1 & 2 & 1.04 & $0.09-12.022$ & 0.9748 \\
\hline Osteoporosis & 1 & 0 & - & - & - \\
\hline Hyperuricemia & 5 & 2 & 6.191 & $1.113-34.444$ & 0.0374 \\
\hline \multicolumn{6}{|l|}{ Medication } \\
\hline NSAIDs & 7 & 1 & 19.526 & $2.252-169.277$ & 0.0070 \\
\hline Antithrombotic agents & 13 & 8 & 5.75 & $1.963-16.840$ & 0.0014 \\
\hline \multicolumn{6}{|c|}{ Multivariate analysis in the cases and controls in the definite group } \\
\hline Hypertension & 15 & 13 & 3.785 & $1.053-13.6$ & 0.0414 \\
\hline Ischemic heart disease & 8 & 4 & 8.28 & $0.502-136.65$ & 0.1395 \\
\hline Cerebrovascular disease & 5 & 2 & 9.964 & $0.677-146.654$ & 0.0938 \\
\hline Hyperuricemia & 5 & 2 & 5.594 & $0.612-51.101$ & 0.1271 \\
\hline NSAIDs & 7 & 1 & 16.766 & $1.529-183.747$ & 0.0210 \\
\hline Antithrombotic agents & 13 & 8 & 1.18 & $0.1-13.936$ & 0.8953 \\
\hline
\end{tabular}

OR, odds ratio; CI, confidence interval; NSAIDs, nonsteroidal anti-inflammatory drugs. 
Table 6. Risk Factors for Diverticular Hemorrhage in the Presumptive Group

\begin{tabular}{|c|c|c|c|c|c|}
\hline Factor & Cases & Controls & OR & $95 \%$ CI & p-value \\
\hline \multicolumn{6}{|c|}{ Univariate analysis in the cases and controls in the presumptive group } \\
\hline Past history & 46 & 106 & & & \\
\hline Diabetes mellitus & 9 & 14 & 1.599 & $0.637-4.012$ & 0.3177 \\
\hline Hypertension & 21 & 28 & 2.34 & $1.135-4.823$ & 0.0212 \\
\hline Hyperlipidemia & 3 & 12 & 0.547 & $0.146-2.037$ & 0.3681 \\
\hline Ischemic heart disease & 9 & 12 & 1.906 & $0.741-4.899$ & 0.1807 \\
\hline Cerebrovascular disease & 9 & 0 & - & - & - \\
\hline Chronic renal disease & 5 & 2 & 6.341 & $1.183-34.017$ & 0.0311 \\
\hline Osteoporosis & 0 & 0 & - & - & - \\
\hline Hyperuricemia & 3 & 2 & 3.628 & $0.585-22.483$ & 0.1661 \\
\hline \multicolumn{6}{|l|}{ Medication } \\
\hline NSAIDs & 12 & 2 & 18.352 & $3.910-86.136$ & 0.0002 \\
\hline Antithrombotic agents & 16 & 12 & 4.178 & $1.779-9.813$ & 0.0011 \\
\hline \multicolumn{6}{|c|}{ Multivariate analysis in the cases and controls in the presumptive group } \\
\hline Hypertension & 21 & 28 & 2.711 & $1.133-6.489$ & 0.0251 \\
\hline Ischemic heart disease & 9 & 12 & 1.317 & $0.423-4.091$ & 0.6341 \\
\hline Chronic renal disease & 5 & 2 & 1.625 & $0.206-12.802$ & 0.6445 \\
\hline Hyperuricemia & 3 & 2 & 0.92 & $0.098-8.602$ & 0.9419 \\
\hline NSAIDs & 12 & 2 & 19.665 & $3.797-101.843$ & 0.0004 \\
\hline Antithrombotic agents & 16 & 12 & 4.828 & $1.603-14.536$ & 0.0051 \\
\hline
\end{tabular}

OR, odds ratio; CI, confidence interval; NSAIDs, nonsteroidal anti-inflammatory drugs.

for the definite group. NSAIDs (OR, 16.766; 95\% CI, 1.5297 to 183.747; $\mathrm{p}=0.021$ ) and hypertension (OR, 3.785; 95\% CI, 1.0532 to $13.6 ; \mathrm{p}=0.04141$ ) were significantly associated in the multivariate analysis. In the presumptive group, the association of NSAIDs (OR, 19.665; 95\% CI, 3.7972 to 101.843; $\mathrm{p}=0.0004$ ), antithrombotic agents (OR, 4.828; 95\% CI, 1.603 to 14.536 ; $\mathrm{p}=0.0051$ ), and hypertension (OR, 2.711; 95\% CI, 1.1327 to 6.489; $p=0.0251$ ) with diverticular hemorrhage was significant in the multivariate analysis (Table 6).

\section{DISCUSSION}

This study identified information about colonic diverticular bleeding. Our results indicated that the current usage of NSAIDs, the presence of cerebrovascular disease, and the presence of hyperuricemia were risk factors for bleeding.

The mechanism for diverticular hemorrhage is not completely understood. However, the bleeding is thought to be a rupture of small arteries, with accumulated endoepithelial damage.,11 Frequently, sclerosis of a vessel might be subject to diverticular bleeding. Diverticular bleeding has been considered to be a result of a rupture of arteriosclerosis. The probable sequence of bleeding is first in injury of physically factors arising within the lumen produce an eccentric damage to the luminal side of the underlying artery. The artery reacts with eccentric intimal thickening. Following persistent or recurrent damage, the initial thickening may become concentric but retains accentuation toward the lumen of the diverticulum. Consequently, progressive segmental weakening of the arterial wall leads to eccentric rupture and hemorrhage. ${ }^{12}$ Arteriosclerosis is commonly accompanies aging, therefore, colonic diverticula and the bleeding frequently occur among older patients. In our study, the average age of patients with diverticular bleeding was 70 years. This fact supported the idea that arteriosclerosis is the main cause of bleeding. However, 16.7\% (12/72) of patients were less than 60 years old. Therefore, we speculated that additional factors might be involved in the vascular frangibility of the diverticula.

The aim of this study is to clarify the significant risk factors for diverticular hemorrhage. Because arteriosclerosis is thought to be the main cause of diverticular bleeding, we matched the patients and controls by age and gender, expecting similar arteriosclerosis levels of arteriosclerosis between them. As a result, we found that the current usage of NSAIDs, as well as the presence of cerebrovascular disease, and hyperuricemia may have an additional influence on the complications of diverticula that lead to vascular damage. In addition, the multivariate analysis of the definite group and presumptive group revealed that the current usage of NSAIDs was considered the most significant risk factor. Previous studies have also reported that NSAIDs were a major cause of diverticular hemorrhage. ${ }^{8,13,14}$ Most 
NSAIDs inhibit prostaglandin synthesis. This might impair the microcirculation and inhibit platelet aggregation, such that bleeding might occur in the presence of diverticular. ${ }^{15}$ In addition, the benefits of selective COX-2 inhibitors for the protection of the gastrointestinal tract have been generally accepted, confirming the inhibitory effect of prostaglandins in gastrointestinal bleeding. Thus, the usage of NSAIDs may increase the vascular frangibility in patients in whom some level of arteriosclerotic change already exists.

Hyperuricemia was also found to be a risk factor for diverticular hemorrhage in the present study. ${ }^{16,17}$ Research has shown that hyperuricemia directly affects diverticular bleeding. Increased uric acid levels promote the oxygenation of low-density lipoprotein cholesterol and facilitate lipid peroxidation, resulting in the increasing the production of oxygen free radicals. Each of these influences is known to plays an essential role in the progression of atherosclerosis. ${ }^{16}$ Consistent with finding of the present study that cerebrovascular disease was a significant risk factor in our study, Okamoto et al. ${ }^{18}$ reported that cardio- or cerebrovascular disease was a significant risk factor for diverticular bleeding. These factors, including hyperuricemia, suggest that systemic metabolic disorders and arteriosclerosis play an important role in diverticular bleeding. A prospective study to investigate the relationship between diverticular bleeding and arteriosclerosis will be needed in the future.

Our study has several limitations. First, a retrospective study includes a potential recall bias. Second, in general, controls should be enrolled from the same source population or study base from which the cases were selected, as was done in our study. However, in a strict sense, not only the similarities in age and sex but also the other factors such as size, location, and numbers of diverticula should have been equalized between the cases and controls. Such detailed analysis may be needed in the future to elucidate the risk factors for diverticular bleeding more precisely.

In conclusion, this study indicates that the current usages of NSAIDs, as well as the presence of cerebrovascular disease and hyperuricemia were significant risk factors for colonic diverticular hemorrhage. The knowledge obtained through this study may give some insight into the diagnostic process for the patients with acute lower gastrointestinal bleeding.

\section{CONFLICTS OF INTEREST}

No potential conflict of interest relevant to this article was reported.

\section{REFERENCES}

1. Stollman N, Raskin JB. Diverticular disease of the colon. Lancet 2004;363:631-639.
2. Painter NS, Burkitt DP. Diverticular disease of the colon: a deficiency disease of Western civilization. Br Med J 1971;2:450-454.

3. Jacobs DO. Clinical practice: diverticulitis. N Engl J Med 2007;357: 2057-2066.

4. Parks TG. Natural history of diverticular disease of the colon. Clin Gastroenterol 1975;4:53-69.

5. Nakaji S, Sugawara K, Saito D, et al. Trends in dietary fiber intake in Japan over the last century. Eur J Nutr 2002;41:222-227.

6. Longstreth GF. Epidemiology and outcome of patients hospitalized with acute lower gastrointestinal hemorrhage: a population-based study. Am J Gastroenterol 1997;92:419-424.

7. Meyers MA, Alonso DR, Gray GF, Baer JW. Pathogenesis of bleeding colonic diverticulosis. Gastroenterology 1976;71:577-583.

8. Yamada A, Sugimoto T, Kondo S, et al. Assessment of the risk factors for colonic diverticular hemorrhage. Dis Colon Rectum 2008; 51:116-120.

9. Jensen DM, Machicado GA, Jutabha R, Kovacs TO. Urgent colonoscopy for the diagnosis and treatment of severe diverticular hemorrhage. N Engl J Med 2000;342:78-82.

10. R Development Core Team. The R project for statistical computing [Internet]. Vienna: R Foundation for Statistical Computing; 2005. Available from: http://www.R-project.org.

11. Hussain A, Mahmood H, Subhas G, El-Hasani S. Complicated diverticular disease of the colon, do we need to change the classical approach, a retrospective study of 110 patients in southeast England. World J Emerg Surg 2008;3:5.

12. Meyers MA, Alonso DR, Baer JW. Pathogenesis of massively bleeding colonic diverticulosis: new observations. AJR Am J Roentgenol 1976;127:901-908.

13. Tsuruoka N, Iwakiri R, Hara M, et al. NSAIDs are a significant risk factor for colonic diverticular hemorrhage in elder patients: evaluation by a case-control study. J Gastroenterol Hepatol 2011;26: 1047-1052.

14. Wilcox CM, Alexander LN, Cotsonis GA, Clark WS. Nonsteroidal antiinflammatory drugs are associated with both upper and lower gastrointestinal bleeding. Dig Dis Sci 1997;42:990-997.

15. Aldoori WH, Giovannucci EL, Rimm EB, Wing AL, Willett WC. Use of acetaminophen and nonsteroidal anti-inflammatory drugs: a prospective study and the risk of symptomatic diverticular disease in men. Arch Fam Med 1998;7:255-260.

16. De Scheerder IK, van de Kraay AM, Lamers JM, Koster JF, de Jong JW, Serruys PW. Myocardial malondialdehyde and uric acid release after short-lasting coronary occlusions during coronary angioplasty: potential mechanisms for free radical generation. Am J Cardiol 1991;68:392-395.

17. Jansen A, Harenberg S, Grenda U, Elsing C. Risk factors for colonic diverticular bleeding: a Westernized community based hospital study. World J Gastroenterol 2009;15:457-461.

18. Okamoto T, Watabe H, Yamada A, Hirata Y, Yoshida H, Koike K. The association between arteriosclerosis related diseases and diverticular bleeding. Int J Colorectal Dis 2012;27:1161-1166. 